\title{
Organography of greenhouse and field accessions of wild Arachis species (section Arachis)
}

Renato Ferraz de Arruda Veiga ${ }^{1}$, Leonardo de Grazia Faria ${ }^{1}$, José Francisco Montenegro Valls², Alessandra Pereira Fávero ${ }^{3 *}$

1.Centro de Recursos Genéticos Vegetais - Instituto Agronômico - Campinas (SP), Brazil.

2.Empresa Brasileira de Pesquisa Agropecuária - Recursos Genéticos e Biotecnologia - Brasília (DF), Brazil.

3.Empresa Brasileira de Pesquisa Agropecuária - Pecuária Sudeste - São Carlos (SP), Brazil.

ABSTRACT: Peanut or groundnut (Arachis hypogaea L.) is a globally important oilseed crop, with important nutritional qualities, and a rich source of amino acids and protein. Another 80 species have been described in the genus Arachis, 64 of which are found in Brazil, and even though their morphology and agronomic traits remain largely unknown, they have been cultivated for forage and for landscaping and have great potential for breeding with cultivated peanut. Thus, the morphological characterization of wild Arachis species is essential for their conservation and use. In this study, we present a morphological and agronomic characterization of 29 diploid accessions of eleven wild Arachis species and one of the tetraploid A. monticola (section Arachis) with $\mathrm{A}$ and $\mathrm{B}$ genomes and determine their intra- and interspecific variability in greenhouse and field conditions. In total, 35 morphological descriptors were developed a priori from greenhouse accessions in the first crop year and used in field accessions in the second crop year. Significant differences in descriptors compiled in the greenhouse and the field support the use of different descriptors for different experimental conditions. PCA analysis showed that the distribution of accessions accorded with the taxonomy of species. The ten morphological descriptors that were important in differentiating section Arachis accessions were seed length, lateral branch length, right apical leaflet length, right apical leaflet width, height and diameter of main stem, branch color, standard petal base color, number of flowers, and presence of bristles on rachis.

Key words: peanut, morphological characterization, descriptors, principal component analysis. 


\section{INTRODUCTION}

The genus Arachis is best known for the cultivated peanut or groundnut (Arachis hypogaea L.), an oil and food crop of high digestibility in natura and high energy value (596 cal/100 $\mathrm{g}$ in average) with seeds rich in amino acids that contain 26 to $31 \%$ of protein (Freire et al. 2009). Interestingly, the genus contains other 80 species native to South America with natural distributions restricted to Argentina, Bolivia, Brazil, Paraguay, and Uruguay, 64 of which are found in Brazil (Hammons 1973; Valls 2005).

Wild Arachis species can be used as animal forage and for landscaping, and have proved useful as an important source of genes for peanut breeding (Oliveira and Valls, 2002; Assis et al. 2013; Santos et al. 2013; Fávero et al. 2015a).

The study of Arachis species is of great importance in Brazil because of the 81 known species in the genus, 64 are native and 48 are unique to the country. Additionally, Brazil is considered the center of origin of the genus Arachis, in the region extending from the states of Mato Grosso do Sul to Goiás (Gregory et al. 1980; Krapovickas and Gregory, 1994; Valls et al. 2013).

The genus Arachis comprises nine sections: Erectoides, Procumbentes, Heteranthae, Caulorrhizae, Extranervosae, Triseminatae, Trierectoides, Rhizomatosae (series Prorhizomatosae and Rhizomatosae), and Arachis, which is of global importance and includes the peanut and other 28 species (Krapovickas and Gregory, 1994; Hilu and Stalker, 1995; Valls and Simpson, 2005).

It was not until the 1980s with collection trips by the Embrapa Genetic Resources and Biotechnonology that the number of populations and new accessions of both cultivated and wild peanut species increased in Brazil. However, little is known about the morphology and agronomic traits of most populations (Peñaloza et al. 2005).

Several wild species have been cultivated for forage and for landscaping, including A. pintoi Krapov. \& W.C. Greg.; A. repens Handro (section Caulorrhizae); A. glabrata Benth. (section Rhizomatosae); A. matiensis Krapov., W.C. Greg. \& C.E. Simpson (section Procumbentes); A. helodes Mart. ex Krapov. \& Rigoni; A. kempff-mercadoi Krapov., W.C. Greg. \& C.E. Simpson; and A. kuhlmannii Krapov. \& W.C. Greg. (section Arachis) (Valls and Pizarro, 1994; Veiga et al. 2003).

Annual and perennial species in the section Arachis, which is considered the best adapted section, have great genetic potential for breeding with cultivated peanut (Simpson and Starr, 2001; Fávero et al. 2006; Michelotto et al. 2015; Fávero et al. 2015b).

Genetic diversity can be increased with the use of plant germplasm from less cultivated modern cultivars, obsolete cultivars, primitive cultivars or local varieties, wild species and special genetic materials, improved varieties that result in special lines, mutants, and others (Breese 1989; Hoyt 1992; Clausen 1997).

Morphologically, the genus Arachis is totally distinct from its closest relatives, because its unusual reproductive system produces geocarpic fruits that originate from aerial flowers with tubular hypanthium, different types of anthers in the same flower, and very delicate seed coat (Singh and Simpson, 1994; Krapovickas and Gregory, 1994; Valls et al. 2013). Stylosanthes is the closest genus to Arachis, with whom it shares fused stipules and the same basic number of chromosomes ( $\mathrm{x}=10)$ (Krapovickas and Gregory, 1994).

The morphological characterization of wild plant species in germplasm banks is essential for the conservation and use of these valuable resources (Veiga et al. 1999).

Germplasm characterization starts with the adoption of a descriptor list. The descriptors can be qualitative traits such as color, habit, texture, presence or absence of characters, and quantitative traits, which vary over a continuous range of phenotypes, including vigor, days until maturity, yield, and height. Even though descriptor lists may be developed by different organizations, they have a similar design (Chapman 1989; IBPGR 1990; Veiga et al. 1992, 2001).

The comparison of morphological descriptors evaluation in greenhouse and field conditions may be very useful to identify the stable characteristics, with same behavior in both environments. With these results, future assay may be done in greenhouse with a lower, but important, number of descriptors. Field assays may be done just for some quantitative descriptors.

In this study, we present a morphological and agronomic characterization of 29 diploid accessions of eleven wild Arachis species and one of the tetraploid A. monticola (section Arachis) with A and B genomes and determine their intra- and interspecific variability in greenhouse and field conditions.

\section{MATERIAL AND METHODS}

We developed morphological descriptors based on (IBPGR 1990) descriptors with modifications to determine the diversity 
and intra- and interspecific variability of accessions under greenhouse and field conditions. The study was conducted over two crop years.

In the first crop year, germplasm provided by the Arachis Germplasm Bank at Embrapa Genetic Resources and Biotechnology was in small amounts and consisted of seeds that had been collected in collection trips and that were kept in the bank, and had to be multiplied. Seeds were sown in Petri dishes with filter paper moistened with distilled water, transplanted into 3-5 aluminum pots following emergence according to the availability of seedlings, and maintained in a greenhouse. The morphological and agronomic descriptors were developed from these greenhouse plants in four replications.

In the second crop year, seeds harvested in the first crop year were sown in plastic bags and four seedlings (replications) were transplanted into the field after 30 days. The same descriptors were determined in the second crop year. Both assays were done based on one way ANOVA.

In both crop years, qualitative, ordinal, and quantitative data were obtained for statistical analysis and assessment of intra- and interspecific variability. The following accessions were examined: A. batizocoi Krapov. \& W.C. Greg. and its mutant (K9484 - Bolivia); A. cruziana Krapov., W.C. Greg. \& C.E.Simpson (Wi1302-2 and Wi1302-3 - Bolivia); A. krapovickasii C.E.Simpson, D.E.Williams, Valls \& I.G.Vargas (Wi1291 - Bolivia); A. hoehnei Krapov. \& W.C. Greg. (V9094, V9146, V13985 and KG30006 - Mato Grosso do Sul); A. schininii Krapov., Valls \& C.E.Simpson (V9923 - Paraguay); A. ipaënsis Krapov. \& W.C. Greg. (KG30076 - Bolivia); A. magna Krapov. \& W.C. Greg. (KG30097 - Bolivia, V13748, V13751, V13760, V13761 and V14707 - Mato Grosso); A. gregoryi C.E.Simpson, Krapov. \& Valls (V14728, V14735, V14739, V14740, V14743, V14753 and V14767 - Mato Grosso); A. monticola Krapov. \& Rigoni (V14165 - Argentina); A. stenosperma Krapov. \& W.C. Greg. (V10229 - São Paulo); A. valida Krapov. \& W.C. Greg. (V9157, V13514, and V13516 - Mato Grosso do Sul); and A. villosa Benth. (V12812 - Uruguay).

The following morphological descriptors were measured with a ruler or a paquimeter or just by the morphological structure observation. They were:

i. Plant characters (in $\mathrm{cm}$ ): height and diameter of main stem (n); diameter and length of secondary branches $(n+1)$; stem pigmentation and peg pigmentation;

ii. Leaf characters: right apical leaflet length and right apical leaflet width; presence of trichomes on rachis, petiole, adaxial and abaxial leaflet surface, free and adnate part of stipule; bristles on: leaflet margins, rachis, petiole, free part of stipule, and adnate part of stipule;

iii. Flower characters: standard petal color, standard petal base color, red pigmentation on standard petal base vertex, number of calyx lobes, presence of calyx bristles, standard petal height, and number of flowers;

iv. Fruit pod characters (in $\mathrm{cm}$ ): length and width of fruit pod, pod beak, and pod reticulation;

V. Seed characters (in $\mathrm{cm}$ ): length and width of seed, and seed color.

Principal component analysis (PCA) was used to determine the distance between greenhouse and field accessions and the distance between each accession in greenhouse and field conditions. SAS software (Release 9.2, SAS Institute Inc., 2011, Cary, NC, USA) was used for obtaining the PCA. The routine of SAS analysis were done to transform quantitative and qualitative data automatically.

The differences in each descriptor between greenhouse and field plants for each accession were compared using the Student's $t$ test for quantitative descriptors and the Fisher's Exact test for qualitative and ordinal descriptors. The correlations between descriptors were calculated using the Pearson's correlation coefficient. Data were considered significant at $\mathrm{p}<0.05$.

\section{RESULTS AND DISCUSSION}

Mean values for each descriptor in greenhouse and field accessions are shown in Table 1.

No significant differences were found in descriptors presence of trichomes on rachis and presence of calyx bristles across accessions (Table 1). Thus, the two descriptors were removed from the PCA because they provided no information about the genetic distance of accessions. The morphological descriptors that exhibited significant differences between greenhouse and field accessions (height and diameter of main stem $[\mathrm{n}]$, length and diameter of secondary branches $[\mathrm{n}+1]$, fruit pod length, fruit pod width, seed length, and seed width; Table 1) are complex quantitative traits, which may explain the difference in these traits between plants grown in the greenhouse and the field. Few descriptors exhibited significant differences between greenhouse and field 
accessions, except for length of secondary branches $(n+1)$, length and width of right apical leaflet and number of flowers, for which the data were significantly higher in field accessions than in greenhouse accessions. This result was expected. Peanut root systems have more space to grow in the field, enabling them to produce larger branches, whereas root growth is limited in greenhouse conditions, resulting in shorter branches. The sun brightness is much higher in field conditions than in greenhouse, which increases the number of flowers observed and also the size of the leaflets to enable photosynthesis in field conditions. The effect of the environment is more pronounced in quantitative than in qualitative traits. In fact, no significant differences in more stable or high-variability traits such as flower color,

Table 1. Morphological descriptors of section Arachis accessions in greenhouse (pot) and field conditions.

\begin{tabular}{|c|c|c|c|c|c|c|c|c|c|c|}
\hline \multirow{2}{*}{$\begin{array}{l}\text { Descriptor/ } \\
\text { accession }\end{array}$} & \multicolumn{2}{|c|}{ V 13748} & \multicolumn{2}{|c|}{ V13751 } & \multicolumn{2}{|c|}{ V13761 } & \multicolumn{2}{|c|}{ V14707 } & \multicolumn{2}{|c|}{ KG 30097} \\
\hline & Pot & Field & Pot & Field & Pot & Field & Pot & Field & Pot & Field \\
\hline A & 12.9 & 7.5 & 6.3 & 11.3 & 9.0 & 9.8 & 5.6 & 12.5 & 18.8 & 8.3 \\
\hline$B$ & 0.4 & 0.6 & 0.4 & 0.4 & 0.5 & 0.6 & 0.3 & 1.0 & 0.5 & 1.5 \\
\hline C & 0.2 & 0.4 & 0.2 & 0.3 & 0.2 & 0.2 & 0.2 & 0.4 & 0.2 & 0.8 \\
\hline D & 40.0 & 90.0 & 65.0 & 142.0 & 55.0 & 163.0 & 45.0 & 164.0 & 70.0 & 146.0 \\
\hline$E$ & 5.2 & 2.3 & 5.3 & 1.9 & 5.7 & 1.6 & 3.8 & 2.1 & 6.0 & 1.4 \\
\hline $\mathrm{F}$ & 3.0 & 1.8 & 2.5 & 1.3 & 2.6 & 1.2 & 2.5 & 1.5 & 3.0 & 0.9 \\
\hline $\mathrm{G}^{\star}$ & 1 & 1 & 0 & 0 & 1 & 1 & 1 & 1 & 0 & 0 \\
\hline $\mathrm{H}^{*}$ & 0 & 0 & 0 & 0 & 0 & 0 & 0 & 0 & 0 & 0 \\
\hline$I^{*}$ & 1 & 1 & 1 & 1 & 1 & 1 & 0 & 0 & 1 & 1 \\
\hline$J^{\star}$ & 1 & 1 & 1 & 1 & 1 & 1 & 1 & 1 & 1 & 1 \\
\hline $\mathrm{K}^{\star}$ & 1 & 1 & 1 & 1 & 1 & 1 & 1 & 1 & 1 & 1 \\
\hline$L^{*}$ & 1 & 1 & 0 & 0 & 1 & 1 & 1 & 1 & 1 & 1 \\
\hline$M^{*}$ & 0 & 0 & 0 & 0 & 1 & 1 & 0 & 0 & 0 & 0 \\
\hline $\mathrm{N}^{*}$ & 0 & 0 & 0 & 0 & 1 & 1 & 0 & 0 & 1 & 1 \\
\hline $\mathrm{O}^{\star}$ & 1 & 1 & 0 & 0 & 1 & 1 & 1 & 1 & 1 & 1 \\
\hline $\mathrm{P}^{\star}$ & 0 & 0 & 0 & 0 & 1 & 1 & 0 & 0 & 0 & 0 \\
\hline $\mathrm{Q}^{\star}$ & 0 & 0 & 0 & 0 & 1 & 1 & 0 & 0 & 0 & 0 \\
\hline $\mathrm{R}^{\star}$ & 0 & 0 & 0 & 0 & 1 & 1 & 0 & 0 & 0 & 0 \\
\hline $\mathrm{S}^{\star}$ & 0 & 0 & 0 & 0 & 1 & 1 & 0 & 0 & 1 & 1 \\
\hline $\mathrm{T}$ & 1.2 & 1.2 & 1.3 & 1.2 & 1.5 & 1.2 & 1.2 & 1.4 & 1.3 & 1.1 \\
\hline U & 0.6 & 0.7 & 0.6 & 0.7 & 0.7 & 0.7 & 0.5 & 0.8 & 0.6 & 0.7 \\
\hline V & 1.1 & 0.9 & 1.3 & 1.0 & 1.4 & 1.0 & 1.3 & 1.0 & 1.2 & 0.9 \\
\hline W & 0.5 & 0.5 & 0.8 & 0.5 & 0.6 & 0.5 & 0.5 & 0.6 & 0.6 & 0.6 \\
\hline$x$ & 3 & 3 & 5 & 5 & 5 & 5 & 3 & 3 & 5 & 5 \\
\hline$Y$ & 5 & 5 & 5 & 5 & 5 & 5 & 3 & 3 & 5 & 5 \\
\hline Z & 3.0 & 3.0 & 4.0 & 4.0 & 5.0 & 5.0 & 3.5 & 3.5 & 4.5 & 4.5 \\
\hline $\mathrm{AA}^{*}$ & 1 & 1 & 1 & 1 & 1 & 1 & 1 & 1 & 1 & 1 \\
\hline$A B^{*}$ & 0 & 0 & 0 & 0 & 0 & 0 & 0 & 0 & 0 & 0 \\
\hline$A C$ & 7 & 14 & 5 & 70 & 5 & 56 & 4 & 85 & 6 & 50 \\
\hline$A D$ & 5 & 5 & 5 & 5 & 4 & 4 & 4 & 4 & 5 & 5 \\
\hline $\mathrm{AE}^{\star \star}$ & 3 & 3 & 3 & 3 & 3 & 3 & 1 & 1 & 3 & 3 \\
\hline $\mathrm{AF}^{\star \star}$ & 3 & 3 & 3 & 3 & 3 & 3 & 3 & 3 & 1 & 1 \\
\hline $\mathrm{AG}^{\star \star \star}$ & 2 & 2 & 2 & 2 & 1 & 1 & 2 & 2 & 1 & 1 \\
\hline
\end{tabular}


anthocyanin pigmentation, and presence of hairs or bristles were observed between greenhouse and field accessions. It is very relevant to identify the descriptors that have the same behavior in distinct environments because it is possible to do new future bioassays in greenhouse and expect that these descriptors would have the same behavior in field conditions. It is known that the characterization in greenhouse conditions is easier than in field, and it is necessary less space, personal and financial resources, etc. So, for a screening, using just the main descriptors in greenhouse conditions is best than using all accessions with a large number of descriptors in field conditions.

The PCA plot for greenhouse and field accessions shows that accessions clustered based on the place where they

Table 1. Continuation...

\begin{tabular}{|c|c|c|c|c|c|c|c|c|c|c|c|c|}
\hline \multirow{2}{*}{$\begin{array}{l}\text { Descriptor/ } \\
\text { accession }\end{array}$} & \multicolumn{2}{|c|}{ V 14728} & \multicolumn{2}{|c|}{ V 14735} & \multicolumn{2}{|c|}{ V 14739} & \multicolumn{2}{|c|}{ V 14740} & \multicolumn{2}{|c|}{ V 14743} & \multicolumn{2}{|c|}{ V 14753} \\
\hline & Pot & Field & Pot & Field & Pot & Field & Pot & Field & Pot & Field & Pot & Field \\
\hline A & 8.0 & 14.5 & 14.8 & 12.8 & 9.5 & 14.3 & 15.6 & 20.3 & 5.3 & 6.5 & 15.3 & 30.8 \\
\hline B & 0.4 & 0.5 & 0.4 & 0.5 & 0.4 & 1.0 & 0.4 & 0.9 & 0.3 & 1.0 & 0.4 & 1.0 \\
\hline C & 0.2 & 0.2 & 0.2 & 0.3 & 0.2 & 0.5 & 0.2 & 0.4 & 0.2 & 0.3 & 0.2 & 0.3 \\
\hline D & 95.0 & 158.0 & 75.0 & 135.0 & 80.0 & 160.0 & 30.0 & 134.0 & 25.0 & 151.0 & 55.0 & 188.0 \\
\hline E & 4.6 & 2.8 & 5.0 & 2.0 & 4.0 & 1.4 & 4.0 & 2.6 & 4.7 & 1.7 & 5.5 & 1.8 \\
\hline $\mathrm{F}$ & 2.6 & 2.0 & 2.5 & 1.6 & 2.5 & 1.3 & 2.0 & 1.1 & 2.5 & 1.2 & 2.7 & 1.2 \\
\hline $\mathrm{G}^{\star}$ & 1 & 1 & 1 & 1 & 1 & 1 & 1 & 1 & 1 & 1 & 1 & 1 \\
\hline $\mathrm{H}^{\star}$ & 0 & 0 & 0 & 0 & 0 & 0 & 0 & 0 & 0 & 0 & 0 & 0 \\
\hline$I^{*}$ & 0 & 0 & 1 & 1 & 1 & 1 & 1 & 1 & 1 & 1 & 1 & 1 \\
\hline$J^{\star}$ & 1 & 1 & 1 & 1 & 1 & 1 & 1 & 1 & 1 & 1 & 1 & 1 \\
\hline$K^{*}$ & 1 & 1 & 1 & 1 & 1 & 1 & 1 & 1 & 1 & 1 & 1 & 1 \\
\hline$L^{*}$ & 1 & 1 & 1 & 1 & 1 & 1 & 1 & 1 & 1 & 1 & 1 & 1 \\
\hline$M^{*}$ & 1 & 1 & 0 & 0 & 0 & 0 & 1 & 1 & 1 & 1 & 1 & 1 \\
\hline $\mathrm{N}^{*}$ & 1 & 1 & 1 & 1 & 1 & 1 & 1 & 1 & 1 & 1 & 1 & 1 \\
\hline $\mathrm{O}^{\star}$ & 0 & 0 & 1 & 1 & 0 & 0 & 1 & 1 & 0 & 0 & 1 & 1 \\
\hline$P^{\star}$ & 1 & 1 & 1 & 1 & 1 & 1 & 1 & 1 & 1 & 1 & 1 & 1 \\
\hline $\mathrm{Q}^{\star}$ & 1 & 1 & 1 & 1 & 1 & 1 & 1 & 1 & 1 & 1 & 1 & 1 \\
\hline $\mathrm{R}^{\star}$ & 0 & 0 & 0 & 0 & 0 & 0 & 0 & 0 & 0 & 0 & 0 & 0 \\
\hline $\mathrm{S}^{\star}$ & 1 & 1 & 0 & 0 & 1 & 1 & 1 & 1 & 1 & 1 & 1 & 1 \\
\hline $\mathrm{T}$ & 1.2 & 1.3 & 1.7 & 1.2 & 1.5 & 1.5 & 1.3 & 1.3 & 1.4 & 1.2 & 1.4 & 1.4 \\
\hline U & 0.6 & 0.8 & 0.8 & 0.7 & 0.7 & 0.8 & 0.6 & 0.8 & 0.6 & 0.7 & 0.7 & 0.8 \\
\hline V & 1.3 & 1.0 & 1.4 & 1.1 & 1.3 & 1.0 & 1.2 & 1.0 & 1.3 & 1.0 & 1.3 & 1.1 \\
\hline W & 0.6 & 0.6 & 0.7 & 0.5 & 0.6 & 0.6 & 0.6 & 0.7 & 0.6 & 0.6 & 0.6 & 0.5 \\
\hline$X$ & 3 & 3 & 5 & 5 & 5 & 5 & 5 & 5 & 3 & 3 & 5 & 5 \\
\hline$Y$ & 3 & 3 & 3 & 3 & 5 & 5 & 5 & 5 & 3 & 3 & 3 & 3 \\
\hline Z & 2.5 & 2.5 & 2.5 & 2.5 & 2.5 & 2.5 & 2.5 & 2.5 & 3.5 & 3.5 & 3.5 & 3.5 \\
\hline $\mathrm{AA}^{*}$ & 1 & 1 & 1 & 1 & 1 & 1 & 1 & 1 & 1 & 1 & 1 & 1 \\
\hline$A B^{\star}$ & 0 & 0 & 0 & 0 & 0 & 0 & 0 & 0 & 0 & 0 & 0 & 0 \\
\hline$A C$ & 6 & 6 & 6 & 6 & 8 & 100 & 8 & 21 & 10 & 15 & 6 & 50 \\
\hline$A D$ & 4 & 4 & 4 & 4 & 4 & 4 & 5 & 5 & 4 & 4 & 5 & 5 \\
\hline$A E^{\star \star}$ & 3 & 3 & 1 & 1 & 3 & 3 & 3 & 3 & 3 & 3 & 1 & 1 \\
\hline $\mathrm{AF}^{\star \star}$ & 3 & 3 & 3 & 3 & 3 & 3 & 3 & 3 & 3 & 3 & 3 & 3 \\
\hline $\mathrm{AG}^{\star \star \star}$ & 1 & 1 & 2 & 2 & 2 & 2 & 2 & 2 & 1 & 1 & 2 & 2 \\
\hline
\end{tabular}


were grown: greenhouse accessions were grouped on the right side of the plot and field accessions were clustered on the left side of the plot (Figure 1). This observation may be explained because the most important descriptors were those that have quantitative behavior. The cumulative sum of the eigenvalues for the two principal components (PC) was $98.9 \%$.
The dispersion of data in Figure 1 can be explained by the fact that the two descriptors that accounted for most of the total variance were length of the secondary branches $(n+1)$ and number of flowers, which exhibited highly significant differences between greenhouse and field accessions. Principal component analysis showed that 10 morphological descriptors were important in explaining the distribution of accessions (in

Table 1. Continuation...

\begin{tabular}{|c|c|c|c|c|c|c|c|c|c|c|}
\hline \multirow{2}{*}{$\begin{array}{l}\text { Descriptor/ } \\
\text { accession }\end{array}$} & \multicolumn{2}{|c|}{ V 14767} & \multicolumn{2}{|c|}{ V 14165} & \multicolumn{2}{|c|}{ V 10229} & \multicolumn{2}{|c|}{ V 13760} & \multicolumn{2}{|c|}{ K 9484 mut } \\
\hline & Pot & Field & Pot & Field & Pot & Field & Pot & Field & Pot & Field \\
\hline A & 9.8 & 11.3 & 11.6 & 5.9 & 7.1 & 8.8 & 7.8 & 10.5 & 31.3 & 16.3 \\
\hline B & 0.5 & 0.6 & 0.3 & 0.4 & 0.3 & 0.4 & 0.2 & 1.3 & 0.4 & 1.0 \\
\hline C & 0.2 & 0.3 & 0.2 & 0.3 & 0.2 & 0.2 & 0.2 & 0.5 & 0.3 & 0.4 \\
\hline $\mathrm{D}$ & 70.0 & 142.0 & 80.0 & 100.0 & 20.0 & 127.0 & 75.0 & 144.0 & 28.0 & 128.0 \\
\hline$E$ & 6.7 & 2.0 & 3.0 & 1.8 & 4.3 & 1.9 & 3.0 & 2.7 & 4.0 & 2.4 \\
\hline$F$ & 3.7 & 1.5 & 1.7 & 1.2 & 1.8 & 1.2 & 2.4 & 1.8 & 3.7 & 2.1 \\
\hline $\mathrm{G}^{\star}$ & 1 & 1 & 0 & 0 & 0 & 0 & 0 & 0 & 1 & 1 \\
\hline $\mathrm{H}^{*}$ & 0 & 0 & 0 & 0 & 0 & 0 & 1 & 1 & 0 & 0 \\
\hline$I^{\star}$ & 1 & 1 & 1 & 1 & 1 & 1 & 1 & 1 & 1 & 1 \\
\hline$J^{\star}$ & 1 & 1 & 1 & 1 & 1 & 1 & 1 & 1 & 1 & 1 \\
\hline $\mathrm{K}^{*}$ & 1 & 1 & 1 & 1 & 1 & 1 & 1 & 1 & 1 & 1 \\
\hline $\mathrm{L}^{*}$ & 1 & 1 & 1 & 1 & 1 & 1 & 1 & 1 & 1 & 1 \\
\hline$M^{\star}$ & 0 & 0 & 0 & 0 & 0 & 0 & 1 & 1 & 1 & 1 \\
\hline $\mathrm{N}^{*}$ & 1 & 1 & 1 & 1 & 0 & 0 & 1 & 1 & 1 & 1 \\
\hline $\mathrm{O}^{\star}$ & 0 & 0 & 1 & 1 & 1 & 1 & 1 & 1 & 0 & 0 \\
\hline $\mathrm{P}^{\star}$ & 0 & 0 & 0 & 0 & 0 & 0 & 1 & 1 & 1 & 1 \\
\hline $\mathrm{Q}^{*}$ & 1 & 1 & 1 & 1 & 1 & 1 & 1 & 1 & 1 & 1 \\
\hline $\mathrm{R}^{\star}$ & 0 & 0 & 0 & 0 & 0 & 0 & 1 & 1 & 1 & 1 \\
\hline $\mathrm{S}^{*}$ & 1 & 1 & 0 & 0 & 0 & 0 & 1 & 1 & 1 & 1 \\
\hline $\mathrm{T}$ & 1.4 & 1.3 & 1.6 & 1.5 & 2.0 & 1.9 & 1.4 & 1.5 & 1.7 & 1.5 \\
\hline U & 0.7 & 0.8 & 0.7 & 0.9 & 0.6 & 0.8 & 0.2 & 0.9 & 0.6 & 0.7 \\
\hline V & 1.4 & 1.1 & 1.3 & 1.2 & 1.7 & 1.6 & 1.2 & 1.2 & 1.3 & 1.4 \\
\hline W & 0.6 & 0.5 & 0.7 & 0.6 & 0.6 & 0.5 & 0.8 & 0.6 & 0.7 & 0.6 \\
\hline$x$ & 5 & 5 & 5 & 5 & 1 & 1 & 3 & 3 & 1 & 1 \\
\hline$Y$ & 3 & 3 & 3 & 3 & 1 & 1 & 3 & 3 & 1 & 1 \\
\hline Z & 4.5 & 4.5 & 3.0 & 3.0 & 2.0 & 2.0 & 2.5 & 2.5 & 2.0 & 2.0 \\
\hline $\mathrm{AA}^{*}$ & 1 & 1 & 1 & 1 & 1 & 1 & 1 & 1 & 1 & 1 \\
\hline$A B^{*}$ & 0 & 0 & 0 & 0 & 0 & 0 & 0 & 0 & 1 & 1 \\
\hline$A C$ & 4 & 15 & 6 & 15 & 10 & 20 & 8 & 20 & 5 & 10 \\
\hline$A D$ & 5 & 5 & 4 & 4 & 4 & 4 & 5 & 5 & 5 & 5 \\
\hline$A E^{\star \star}$ & 3 & 3 & 3 & 3 & 3 & 3 & 3 & 3 & 1 & 1 \\
\hline$A F^{\star \star}$ & 3 & 3 & 1 & 1 & 1 & 1 & 1 & 1 & 1 & 1 \\
\hline$A G^{\star \star \star}$ & 2 & 2 & 2 & 2 & 1 & 1 & 1 & 1 & 2 & 2 \\
\hline
\end{tabular}


order of importance): length $(n+1)$, number of flowers, height of main stem (n), right apical leaflet length, right apical leaflet width, diameter $(\mathrm{n})$, seed length, anthocyanin pigmentation of branches, bristles on rachis, and standard petal base color. In fact, several studies have shown that characters such as branch length, leaflet length, and leaflet width are important in discriminating accessions of Arachis species (Veiga et al. 2001; Castro et al. 2007; Fávero et al. 2015a, Fávero et al. 2015b). For instance, Costa $(2012)^{4}$ examined six floral structures in several wild Arachis species, all of which were also evaluated in this study except for A. villosa and A. ipaënsis, and found significant differences in all floral structures for all species.

Table 1. Continuation...

\begin{tabular}{|c|c|c|c|c|c|c|c|c|c|c|c|c|}
\hline \multirow{2}{*}{$\begin{array}{l}\text { Descriptor/ } \\
\text { accession }\end{array}$} & \multicolumn{2}{|c|}{ K 9484} & \multicolumn{2}{|c|}{ V 12812} & \multicolumn{2}{|c|}{ Wi 1302-2 } & \multicolumn{2}{|c|}{ Wi 1302-3 } & \multicolumn{2}{|c|}{ Wi 1291} & \multicolumn{2}{|c|}{ V 9157} \\
\hline & Pot & Field & Pot & Field & Pot & Field & Pot & Field & Pot & Field & Pot & Field \\
\hline$A$ & 22.5 & 15.3 & 7.3 & 5.8 & 8.3 & 17.0 & 8.9 & 14.0 & 29.8 & 20.5 & 13.3 & 12.3 \\
\hline B & 0.4 & 0.5 & 0.2 & 0.5 & 0.4 & 0.6 & 0.4 & 1.3 & 0.4 & 0.6 & 0.6 & 0.5 \\
\hline C & 0.3 & 0.3 & 0.1 & 0.2 & 0.2 & 0.2 & 0.2 & 0.4 & 0.2 & 0.3 & 0.2 & 0.3 \\
\hline $\mathrm{D}$ & 45.0 & 200.0 & 7.0 & 121.0 & 20.0 & 200.0 & 50.0 & 164.0 & 75.0 & 250.0 & 40.0 & 88.0 \\
\hline$E$ & 5.0 & 2.5 & 4.3 & 1.5 & 5.0 & 2.0 & 5.5 & 1.8 & 6.0 & 2.8 & 5.0 & 3.0 \\
\hline$F$ & 3.8 & 2.1 & 1.9 & 1.0 & 2.1 & 1.3 & 2.5 & 1.2 & 4.0 & 1.4 & 1.7 & 1.6 \\
\hline $\mathrm{G}^{\star}$ & 1 & 1 & 1 & 1 & 1 & 1 & 1 & 1 & 1 & 1 & 0 & 0 \\
\hline $\mathrm{H}^{*}$ & 0 & 0 & 0 & 0 & 0 & 0 & 0 & 0 & 0 & 0 & 0 & 0 \\
\hline$I^{*}$ & 1 & 1 & 1 & 1 & 1 & 1 & 1 & 1 & 1 & 1 & 0 & 0 \\
\hline$J^{*}$ & 1 & 1 & 1 & 1 & 1 & 1 & 1 & 1 & 0 & 0 & 0 & 0 \\
\hline$K^{*}$ & 1 & 1 & 1 & 1 & 1 & 1 & 1 & 1 & 1 & 1 & 1 & 1 \\
\hline$L^{*}$ & 1 & 1 & 1 & 1 & 1 & 1 & 1 & 1 & 1 & 1 & 1 & 1 \\
\hline$M^{*}$ & 1 & 1 & 0 & 0 & 1 & 1 & 1 & 1 & 0 & 0 & 0 & 0 \\
\hline $\mathrm{N}^{*}$ & 1 & 1 & 1 & 1 & 1 & 1 & 1 & 1 & 1 & 1 & 1 & 1 \\
\hline $\mathrm{O}^{*}$ & 0 & 0 & 1 & 1 & 1 & 1 & 1 & 1 & 1 & 1 & 1 & 1 \\
\hline $\mathrm{P}^{\star}$ & 1 & 1 & 0 & 0 & 1 & 1 & 1 & 1 & 1 & 1 & 0 & 0 \\
\hline $\mathrm{Q}^{*}$ & 1 & 1 & 1 & 1 & 1 & 1 & 1 & 1 & 1 & 1 & 1 & 1 \\
\hline $\mathrm{R}^{\star}$ & 1 & 1 & 0 & 0 & 1 & 1 & 1 & 1 & 0 & 0 & 0 & 0 \\
\hline $\mathrm{S}^{\star}$ & 1 & 1 & 0 & 0 & 1 & 1 & 1 & 1 & 1 & 1 & 0 & 0 \\
\hline $\mathrm{T}$ & 1.4 & 1.4 & 1.6 & 1.4 & 1.5 & 1.2 & 1.3 & 1.3 & 1.5 & 1.1 & 1.3 & 1.3 \\
\hline $\mathrm{U}$ & 0.6 & 0.7 & 0.6 & 0.7 & 0.7 & 0.7 & 0.6 & 0.7 & 0.8 & 0.8 & 0.6 & 0.7 \\
\hline V & 1.3 & 1.1 & 1.4 & 1.1 & 1.4 & 1.1 & 1.2 & 1.1 & 1.6 & 1.0 & 1.3 & 1.2 \\
\hline W & 0.6 & 0.6 & 0.7 & 0.6 & 0.6 & 0.6 & 0.5 & 0.6 & 0.8 & 0.7 & 0.6 & 0.6 \\
\hline$x$ & 1 & 1 & 3 & 3 & 3 & 3 & 1 & 1 & 3 & 3 & 5 & 5 \\
\hline$Y$ & 1 & 1 & 1 & 1 & 1 & 1 & 3 & 3 & 3 & 3 & 3 & 3 \\
\hline Z & 2.0 & 2.0 & 1.5 & 1.5 & 2.0 & 2.0 & 2.0 & 2.0 & 2.0 & 2.0 & 4.5 & 4.5 \\
\hline $\mathrm{AA}^{*}$ & 1 & 1 & 1 & 1 & 1 & 1 & 1 & 1 & 1 & 1 & 1 & 1 \\
\hline$A B^{\star}$ & 1 & 1 & 0 & 0 & 1 & 1 & 1 & 1 & 1 & 1 & 0 & 0 \\
\hline$A C$ & 6 & 18 & 7 & 30 & 8 & 18 & 5 & 10 & 7 & 10 & 6 & 5 \\
\hline$A D$ & 4 & 4 & 5 & 5 & 5 & 5 & 5 & 5 & 4 & 4 & 4 & 4 \\
\hline$A E^{\star \star}$ & 3 & 3 & 1 & 1 & 1 & 1 & 1 & 1 & 3 & 3 & 3 & 3 \\
\hline$A F^{\star \star}$ & 1 & 1 & 1 & 1 & 3 & 3 & 3 & 3 & 3 & 3 & 3 & 3 \\
\hline$A G^{\star \star \star}$ & 1 & 1 & 1 & 1 & 2 & 2 & 2 & 2 & 2 & 2 & 2 & 2 \\
\hline
\end{tabular}

${ }^{4}$ Costa, L.C. (2012). Biologia floral de espécies do gênero Arachis L. (Fabaceae-Papilonoideae), com ênfase em aspectos da morfologia floral e na anatomia de ovários. [Tese]. Brasília: Universidade de Brasília. 
In Figure 1, A. magna accessions were scattered across the plot within each group (greenhouse and field accessions), showing high variability of morphological characters within the species, whereas accessions of other species such $A$. hoehnei and A. valida were closer in the plot. The single A. villosa accession examined was situated close to the A. stenosperma accession. This result was expected because both species have A genome
(Fernández and Krapovickas, 1994). Similarly, A. ipaënsis was close to $A$. valida. This result supports molecular marker studies that show that $A$. valida is close to $A$. ipaënsis and has $B$ genome (Robledo and Seijo, 2010; Custodio et al. 2013).

The Pearson's correlation coefficients for all descriptors are shown in Table 2. The most interesting positive correlations with high correlation coefficients $\left(r_{p}>0.6\right)$

Table 1. Continuation...

\begin{tabular}{|c|c|c|c|c|c|c|c|c|}
\hline \multirow{2}{*}{$\begin{array}{l}\text { Descriptor/ } \\
\text { accession }\end{array}$} & \multicolumn{2}{|c|}{ V 13514} & \multicolumn{2}{|c|}{ V 13516} & \multicolumn{2}{|c|}{ V 9094} & \multicolumn{2}{|c|}{ V 9146} \\
\hline & Por & Field & Por & Field & Por & Field & Por & Field \\
\hline$A$ & 8.5 & 9.3 & 5.9 & 17.3 & 4.6 & 7.3 & 5.9 & 16.5 \\
\hline B & 0.3 & 1.3 & 0.3 & 1.2 & 0.2 & 0.8 & 0.2 & 0.7 \\
\hline C & 0.2 & 0.4 & 0.2 & 0.7 & 0.2 & 0.4 & 0.2 & 0.3 \\
\hline D & 55.0 & 76.0 & 25.0 & 88.0 & 10.0 & 126.0 & 6.0 & 110.0 \\
\hline$E$ & 4.0 & 3.3 & 3.0 & 2.0 & 3.6 & 2.2 & 5.0 & 2.3 \\
\hline $\mathrm{F}$ & 1.5 & 1.8 & 1.8 & 1.5 & 1.8 & 1.3 & 2.0 & 1.0 \\
\hline $\mathrm{G}^{\star}$ & 0 & 0 & 1 & 1 & 1 & 1 & 1 & 1 \\
\hline $\mathrm{H}^{*}$ & 0 & 0 & 0 & 0 & 1 & 1 & 1 & 1 \\
\hline$I^{\star}$ & 1 & 1 & 0 & 0 & 0 & 0 & 0 & 0 \\
\hline$J^{*}$ & 1 & 1 & 0 & 0 & 0 & 0 & 1 & 1 \\
\hline $\mathrm{K}^{*}$ & 1 & 1 & 1 & 1 & 1 & 1 & 1 & 1 \\
\hline$L^{*}$ & 1 & 1 & 1 & 1 & 1 & 1 & 1 & 1 \\
\hline$M^{*}$ & 0 & 0 & 1 & 1 & 1 & 1 & 1 & 1 \\
\hline $\mathrm{N}^{*}$ & 1 & 1 & 1 & 1 & 1 & 1 & 1 & 1 \\
\hline $\mathrm{O}^{*}$ & 0 & 0 & 0 & 0 & 1 & 1 & 1 & 1 \\
\hline $\mathrm{P}^{*}$ & 0 & 0 & 0 & 0 & 1 & 1 & 1 & 1 \\
\hline $\mathrm{Q}^{*}$ & 1 & 1 & 1 & 1 & 1 & 1 & 1 & 1 \\
\hline$R^{*}$ & 0 & 0 & 0 & 0 & 1 & 1 & 0 & 0 \\
\hline $\mathrm{S}^{*}$ & 0 & 0 & 1 & 1 & 1 & 1 & 1 & 1 \\
\hline $\mathrm{T}$ & 1.7 & 1.5 & 1.3 & 1.3 & 1.2 & 1.3 & 1.1 & 1.1 \\
\hline$U$ & 0.8 & 0.9 & 0.7 & 0.8 & 0.6 & 0.7 & 0.6 & 0.7 \\
\hline V & 1.4 & 1.3 & 1.2 & 1.1 & 1.2 & 1.1 & 1.4 & 0.9 \\
\hline W & 0.7 & 0.6 & 0.6 & 0.6 & 0.5 & 0.5 & 0.6 & 0.5 \\
\hline$x$ & 5 & 5 & 5 & 5 & 3 & 3 & 3 & 3 \\
\hline$Y$ & 5 & 5 & 5 & 5 & 3 & 3 & 3 & 3 \\
\hline Z & 3.0 & 3.0 & 4.5 & 4.5 & 2.0 & 2.0 & 3.0 & 3.0 \\
\hline $\mathrm{AA}^{*}$ & 1 & 1 & 1 & 1 & 1 & 1 & 1 & 1 \\
\hline$A B^{\star}$ & 0 & 0 & 0 & 0 & 0 & 0 & 0 & 0 \\
\hline$A C$ & 7 & 10 & 5 & 5 & 6 & 30 & 5 & 30 \\
\hline$A D$ & 4 & 4 & 4 & 4 & 5 & 5 & 5 & 5 \\
\hline$A E^{\star \star}$ & 3 & 3 & 3 & 3 & 3 & 3 & 3 & 3 \\
\hline $\mathrm{AF}^{\star \star}$ & 3 & 3 & 3 & 3 & 1 & 1 & 1 & 1 \\
\hline$A G^{\star \star \star}$ & 1 & 1 & 1 & 1 & 2 & 2 & 1 & 1 \\
\hline
\end{tabular}


Table 1. Continuation...

\begin{tabular}{|c|c|c|c|c|c|c|c|c|}
\hline \multirow{2}{*}{$\begin{array}{l}\text { Descriptor/ } \\
\text { accession }\end{array}$} & \multicolumn{2}{|c|}{ V 13985} & \multicolumn{2}{|c|}{ KG 30006} & \multicolumn{2}{|c|}{ V 9923} & \multicolumn{2}{|c|}{ KG 30076} \\
\hline & Por & Field & Por & Field & Por & Field & Por & Field \\
\hline$A$ & 9.0 & 17.3 & 7.5 & 9.0 & 3.8 & 13.0 & 4.6 & 16.8 \\
\hline B & 0.3 & 0.6 & 0.2 & 0.3 & 0.3 & 0.4 & 0.2 & 0.4 \\
\hline$C$ & 0.2 & 0.4 & 0.2 & 0.2 & 0.2 & 0.3 & 0.2 & 0.3 \\
\hline D & 20.0 & 140.0 & 25.0 & 140.0 & 40.0 & 145.0 & 12.0 & 70.0 \\
\hline$E$ & 4.5 & 2.4 & 3.6 & 1.7 & 4.2 & 2.5 & 2.5 & 2.2 \\
\hline $\mathrm{F}$ & 1.5 & 1.2 & 1.5 & 1.0 & 2.1 & 1.5 & 1.9 & 1.5 \\
\hline $\mathrm{G}^{*}$ & 1 & 1 & 1 & 1 & 1 & 1 & 0 & 0 \\
\hline $\mathrm{H}^{*}$ & 1 & 1 & 1 & 1 & 1 & 1 & 0 & 0 \\
\hline$I^{*}$ & 0 & 0 & 0 & 0 & 0 & 1 & 0 & 1 \\
\hline$J^{\star}$ & 0 & 0 & 1 & 1 & 1 & 1 & 1 & 1 \\
\hline $\mathrm{K}^{\star}$ & 1 & 1 & 1 & 1 & 1 & 1 & 1 & 1 \\
\hline$L^{*}$ & 1 & 1 & 1 & 1 & 1 & 1 & 1 & 1 \\
\hline$M^{*}$ & 1 & 1 & 1 & 1 & 0 & 0 & 0 & 0 \\
\hline $\mathrm{N}^{*}$ & 1 & 1 & 1 & 1 & 1 & 1 & 0 & 0 \\
\hline $\mathrm{O}^{*}$ & 1 & 1 & 0 & 0 & 1 & 1 & 0 & 0 \\
\hline$P^{\star}$ & 1 & 1 & 1 & 1 & 1 & 1 & 0 & 0 \\
\hline $\mathrm{Q}^{*}$ & 1 & 1 & 1 & 1 & 1 & 1 & 1 & 1 \\
\hline $\mathrm{R}^{\star}$ & 0 & 0 & 0 & 0 & 0 & 0 & 0 & 0 \\
\hline $\mathrm{S}^{\star}$ & 1 & 1 & 0 & 0 & 1 & 1 & 0 & 0 \\
\hline$T$ & 1.0 & 1.2 & 1.4 & 1.4 & 1.5 & 1.4 & 1.2 & 1.4 \\
\hline$U$ & 0.6 & 0.7 & 0.5 & 0.7 & 0.6 & 0.7 & 0.6 & 0.8 \\
\hline $\mathrm{V}$ & 1.2 & 1.1 & 1.2 & 1.2 & 1.3 & 1.1 & 1.1 & 1.1 \\
\hline W & 0.6 & 0.5 & 0.5 & 0.6 & 0.6 & 0.6 & 0.5 & 0.7 \\
\hline$x$ & 3 & 3 & 3 & 3 & 1 & 1 & 5 & 5 \\
\hline$Y$ & 3 & 3 & 3 & 3 & 3 & 3 & 5 & 5 \\
\hline Z & 3.0 & 3.0 & 4.0 & 4.0 & 2.0 & 2.0 & 1.5 & 1.5 \\
\hline$A A^{*}$ & 1 & 1 & 1 & 1 & 1 & 1 & 1 & 1 \\
\hline$A B^{*}$ & 0 & 0 & 0 & 0 & 0 & 0 & 0 & 0 \\
\hline$A C$ & 9 & 35 & 12 & 24 & 6 & 18 & 3 & 18 \\
\hline$A D$ & 5 & 5 & 4 & 4 & 5 & 5 & 5 & 5 \\
\hline$A E^{\star \star}$ & 3 & 3 & 3 & 3 & 3 & 3 & 3 & 3 \\
\hline $\mathrm{AF}^{\star \star}$ & 1 & 1 & 1 & 1 & 1 & 1 & 3 & 3 \\
\hline$A G^{\star \star \star}$ & 2 & 2 & 2 & 2 & 1 & 1 & 1 & 1 \\
\hline
\end{tabular}

Values in bold are significant at $\mathrm{p}<0.05$ (Student's t test or Fisher's Exact test). $0=$ Absent; $1=$ Present; Pod reticulation: $1=$ None, $3=$ Moderate, 5 = Prominent; Pod beak: 1 = Slight, $3=$ Moderate, $5=$ Proeminent; ${ }^{\star \star}$ : 1 = yellowish, $3=$ Orange, $5=$ Reddish; ${ }^{\star \star \star}: 1=$ United, $2=$ Free; A = Height of main stem $(n)(\mathrm{cm}) ; B=$ Diameter of main stem $(n)(\mathrm{cm}) ; C=$ Diameter of secondary branch $(n+1)(\mathrm{cm}) ; D=L$ Length of secondary branch $(n+1)(\mathrm{cm})$; $\mathrm{E}=$ Right apical leaflet length $(\mathrm{cm}) ; \mathrm{F}=$ Right apical leaflet width $(\mathrm{cm}) ; \mathrm{G}=$ Presence of trichomes on adaxial leaflet surface; $\mathrm{H}=\mathrm{Presence}$ of trichomes on abaxial leaflet surface; I = Anthocyanin pigmentation of branches; $\mathrm{J}=$ Anthocyanin pigmentation of pegs; $\mathrm{K}=\mathrm{Presence}$ of trichomes on rachis; $\mathrm{L}=$ Presence of trichomes on petiole; $\mathrm{M}=$ Presence of trichomes on free part of stipule; $\mathrm{N}=$ Presence of trichomes on stipule base; $\mathrm{O}=\mathrm{Bristles}$ on leaflet margins; $\mathrm{P}=$ Bristles on rachis; $\mathrm{Q}=$ Bristles on petiole; $\mathrm{R}=$ Bristles on free part of stipule; $\mathrm{S}=\mathrm{Bristles}$ on stipule base; $\mathrm{T}=\mathrm{Fruit}$ pod length $(\mathrm{cm}) ; \mathrm{U}=$ Fruit pod width $(\mathrm{cm}) ; \mathrm{V}=$ Seed length $(\mathrm{cm}) ; \mathrm{W}=$ Seed width $(\mathrm{cm}) ; X=$ Pod reticulation; $Y=$ Pod beak; $Z=S t a n d a r d ~ p e t a l ~ h e i g h t ~(\mathrm{~cm}) ; A A=$

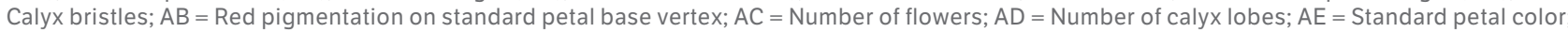
$\mathrm{AF}=$ Standard petal base color; $\mathrm{AG}=$ Calyx lobes . 


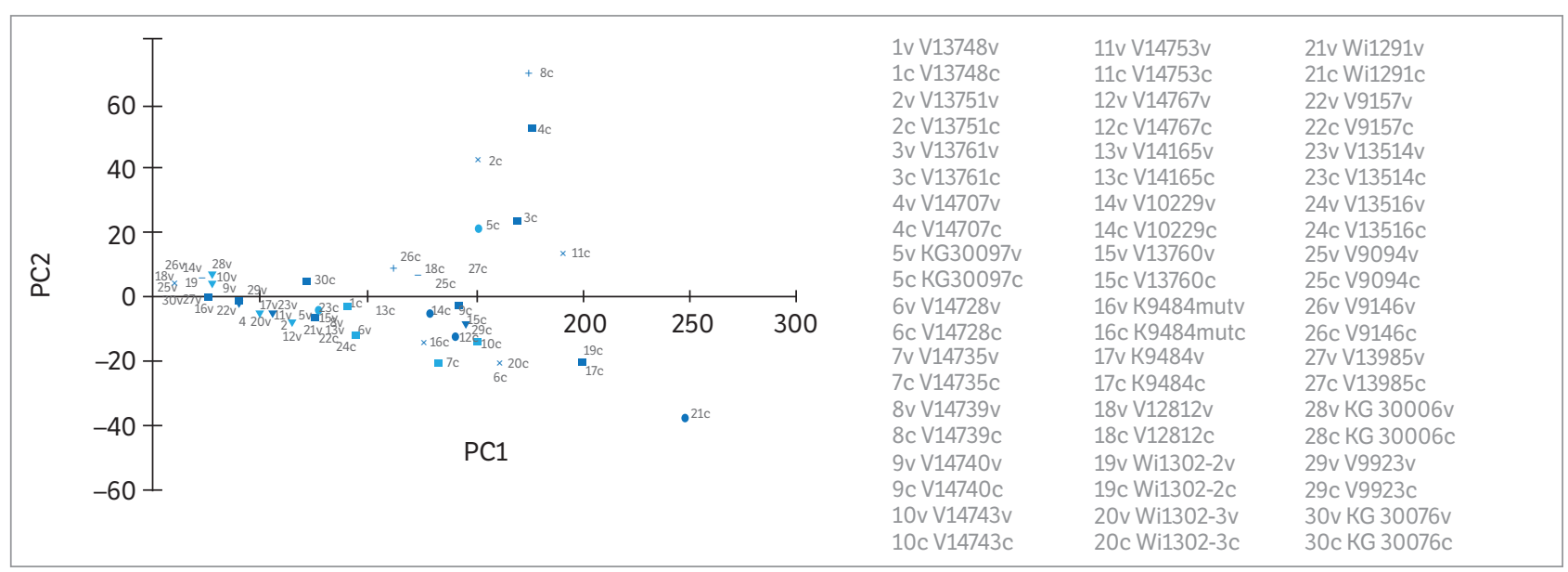

Figure 1. Principal component analysis of $\mathrm{PC} 1 \times \mathrm{PC} 2.2 \mathrm{D}$ plot showing the distribution of greenhouse (accessions followed by the letter $\mathrm{v}$ ) and field (accessions followed by the letter $\mathrm{c}$ ) accessions.

include those between: right apical leaflet length and right apical leaflet width; diameter of main stem and diameter of secondary branch $(n+1)$; presence of trichomes on adnate part of stipule and bristles on adnate part of stipule; presence of trichomes on stipule base and bristles on stipule base; bristles on rachis and bristles on the adnate part of stipule; fruit pod length and seed length; and pod reticulation and pod beak.

There was a slight tendency of more intense pigmentation of branches in greenhouse accessions than in field accessions, but the differences were not significant $(\mathrm{p}=\mathrm{ns}$, all).

Figure 2 shows the principal component analysis for field (Figure 2a) and greenhouse (Figure 2b) accessions. There was a tendency for the clustering of accessions of the same species. The following descriptors were important in discriminating greenhouse accessions (in order of importance): length $(n+1)$, height of main stem, seed width, pod beak, right apical leaflet width, right apical leaflet length, number of flowers, standard petal base color, standard petal height, and anthocyanin pigmentation of branches. The cumulative sum of the eigenvalues for the two principal components was $98.1 \%$. The following descriptors were important in discriminating field accessions (in order of importance): length $(n+1)$, number of flowers, height of main stem, pod beak, seed width, bristles on rachis, bristles on stipule base, standard petal color, reddish pigmentation on standard petal base vertex, and hairiness of abaxial leaflet surface. The cumulative sum of the eigenvalues for the two principal components was $98.5 \%$. Thus, six out of 10 descriptors were important in discriminating greenhouse and field accessions: length $(n+1)$, number of flowers, height of main stem, pod beak, seed width, and standard petal color.

Closely related species such as A. valida and A. magna were also close when greenhouse and field accessions were analyzed separately (Figure 2a,b). Similarly, A genome

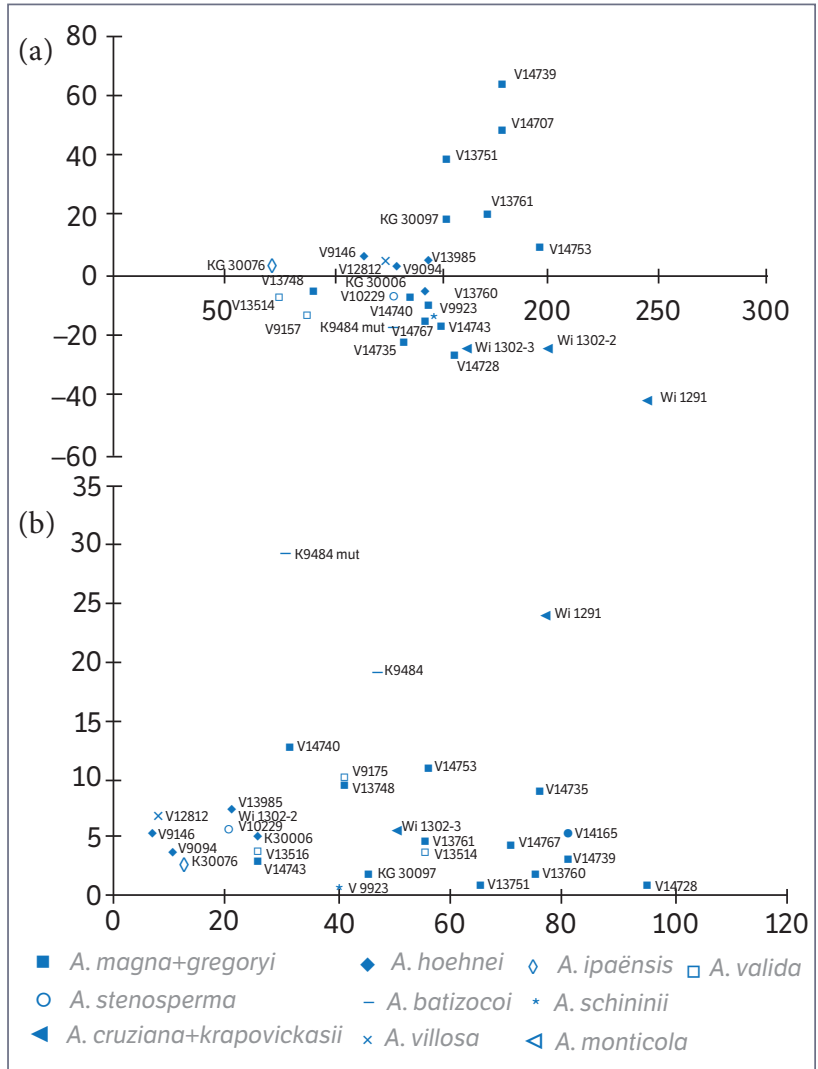

Figure 2. PCA of Arachis accessions using 31 morphological descriptors. (a) Field accessions; (b) greenhouse accessions. 


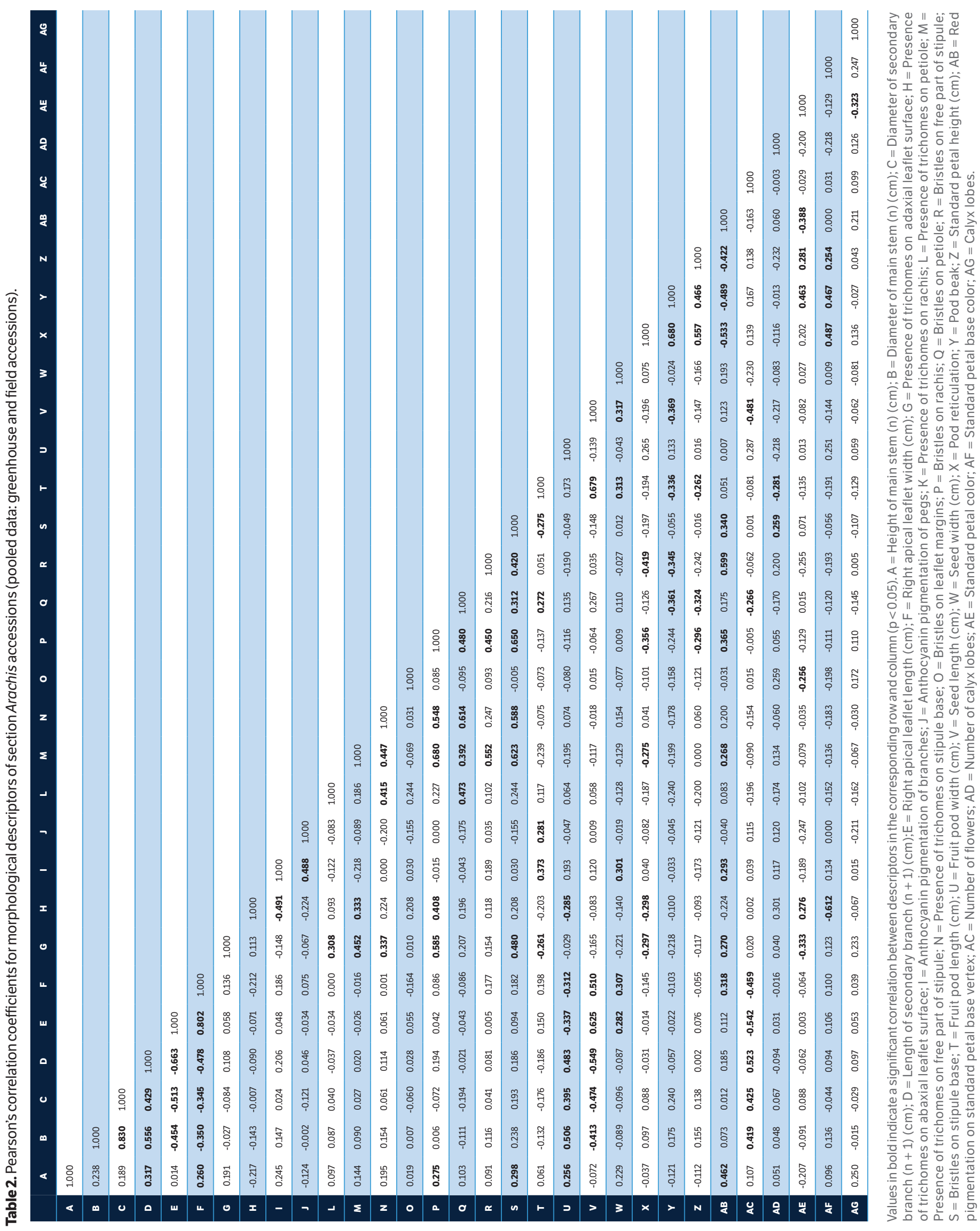


species such as A. schininii, A. stenosperma, and A. hoehnei were also close. Moreover, accessions of the same species clustered more closely in field conditions (Figure 2a) than in greenhouse conditions (Figure 2b).

\section{CONCLUSION}

The majority of the descriptors had the same behavior in distinct environments. So, it will be possible to do new future bioassays in greenhouse and expect that these descriptors would have the same behavior in field conditions. Significant differences are detected between data collected in the greenhouse and the field in quantitative descriptors. Thus, in these cases, morphological and agronomic descriptors should be used specifically in the conditions for which they were developed. In this study, regardless of environment (greenhouse or field), the 10 morphological descriptors that are important in differentiating section Arachis accessions are seed length, lateral branch length, right apical leaflet length, right apical leaflet width, height and diameter of main stem, branch color, standard petal base color, number of flowers, and presence of bristles on rachis.

\section{ACKNOWLEDGMENTS}

The authors thank Instituto Agronômico de Campinas, National Council for Scientific and Technological Development (CNPq) and Brazilian Agricultural Research Corporation (Embrapa) for their financial support.

\section{REFERENCES}

Assis, G. M. L., Valentim, J. F. and Andrade, C. M. S. (2013). BRS Mandobi: a new forage peanut cultivar propagated by seeds for the tropics. Tropical Grasslands - Forrajes Tropicales, 1, 39-41. https://doi.org/10.17138/tgft(1)39-41.

Breese, L. (1989). Multiplication and Regeneration of Germplasm. In H. T. Stalker and C. Chapman. (Eds.), IBPGR Training Courses: Lecture Series, 2. Scientific management of germplasm: characterization, evaluation and enhancement (p. 17-21). Rome: International Board for Plant Genetic Resources.

Castro, C. M., Wagner, C. M., Valls, J. F. M. and Karia, C. T. (2007). Morphological traits as variety descriptors of Arachis pintoi. Crop Breeding and Applied Biotechnology, 7, 279-287. https://doi.org/10.12702/1984-7033.v07n03a07.

Chapman, C. (1989). Principles of Germplasm Evaluation. In: H. T. Stalker and C. Chapman (Eds.), IBPGR Training Courses: lecture Series, 2. Scientific management of germplasm: characterization, evaluation and enhancement (p. 55-63). Rome: International Board for Plant Genetic Resources.

Clausen, A. M. (1997). La red. Informe Especial Campo y Tecnología-Agrobiodiversidad-Conservación y Utilización Sustentable. (p .4-6). Buenos Aires: INTA.
Custodio, A. R., Seijo, G. and Valls, J. F. M. (2013) Characterization of Brazilian accessions of wild Arachis species of section Arachis (Fabaceae) using heterochromatin detection and fluorescence in situ hybridization (FISH). Genetics and Molecular Biology, 36, 364370. https://doi.org/10.1590/s1415-47572013000300011.

Fávero, A. P.; Simpson, C. E., Valls, J. F. M. and Vello, N. A. (2006). Study of the evolution of cultivated peanut through crossability studies among Arachis ipaensis, A. duranensis, and $A$. hypogaea. Crop Science, 46, 1546-1552. https://doi.org/10.2135/ cropsci2005.09-0331.

Fávero, A. P., Pádua, J. G., Gimenes, M. A., Godoy, I. J., Moretzsohn, M. C. and Michelotto, M. D. (2015b). New hybrids from peanut (Arachis hypogaea L.) and synthetic amphidiploid crosses show promise in increasing pest and disease tolerance. Genetics and Molecular Research, 14, 16694-16703. https://doi.org/10.4238/2015. december.11.17.

Fávero, A. P., Santos, R. F., Simpson, C. E., Valls, J. F. M. and Vello, N. A. (2015a). Successful crosses between fungal-resistant wild species of Arachis (Section Arachis) and Arachis hypogaea. Genetics and Molecular Biology, 38,353-365. https://doi.org/10.1590/ s1415-475738320140376.

Fernández, A. and Krapovickas, A. (1994). Cromosomas y evolución en Arachis (Leguminosae). Bonplandia, 8, 187-220. 
Freire, R. M. M., Santos, R. C., Silva, A. C. and Lima, L. M. (2009). Propriedades nutricionais e processamento. In R. C. Santos, R. M. M. Freire and T. M. F. Suassuna. Amendoim: o produtor pergunta, a Embrapa responde (p. 179-200). Brasília: Embrapa Informação Tecnológica.

Gregory, W. C., Krapovickas, A., and Gregory, M. P. (1980). Structure, variation, evolution, and classification in Arachis. In R. J. Summerfield, Bunting, A. H. Advances in Legume Science (p. 469-481). Kew: Royal Botanic Gardens.

Hammons, R. O. (1973). Early history and origin of the peanut. In C. T. Wilson (Ed.), Peanuts - Culture and Uses (p. 17-45). Stillwater, Oklahoma: American Peanut Research and Education Association.

Hilu, K. W. and Stalker, H. T. (1995). Genetic relationships between peanutand wild species of Arachis sect. Arachis (Fabaceae): evidence from RAPDs. Plant Systematics and Evolution, 198,167-178. https:// doi.org/10.1007/bf00984735.

Hoyt, E. (1992). Conservação dos parentes silvestres das plantas cultivadas (p. 52). Wilmington: IBPGR/IUCN/ WWF.

International Board for Plant Genetic Resources (1990). Preliminary descriptors for Arachis - International Crop Network Series. 2. Report or a Workshop on the Genetic Resources of Wild Arachis Species. International Board for Plant Genetic Resources, Rome.

Krapovickas, A. and Gregory, W. C. (1994). Taxonomía del gênero Arachis (Leguminosae). Bonplandia, 8, 1-186.

Michelotto, M. D., Barioni, W., Resende, M. D. V., Godoy, I. J., Leonardecz, E. and Fávero, A. P. (2015). Identification of fungus resistant wild accessions and interspecific hybrids of the genus Arachis. PLoS One, 10, e0128811. https://doi.org/10.1371/journal.pone.0128811.

Oliveira, M. A. P. and Valls, J. F. M. (2002). Produção de híbridos de amendoim forrageiro por meio de hibridação artificial. Pesquisa Agropecuária Brasileira, 37, 885-888. https://doi.org/10.1590/ s0100-204x2002000600019.

Peñaloza, A. P. S, Valls, J. F. M. and Silva, G. P. (2005). Coleta de germoplasma de espécies silvestres de amendoim (Arachis L. Leguminosae). In B.MT.Walter and T.B. Cavalcanti (Eds.), Fundamentos para a coleta de germoplasma vegetal (p. 308-325), Brasília: Embrapa Recursos Genéticos e Biotecnologia.

Robledo, G. and Seijo, G. (2010). Species relationships among the wild B genome of Arachis species (Section Arachis) based on FISH mapping of rDNA lociand heterochromatin detection: a new proposal for genomearrangement. Theoretical and Applied Genetics, 121,10331046. http://dx.doi.org/10.1007/s00122-010-1369-7.

Santos, J. F., Godoy, I. J., Michelotto, M. D. and Fávero, A. P. (2013). Resistência à mancha preta e qualidade agronômica de plantas RC1F2 de cruzamentos do híbrido anfidiplóide (A. ipaensis $\times$ A. duranensis) como amendoim cultivado (Arachis hypogaea L.). Bioscience Journal, 29,280-287. https://doi.org/10.1590/s0006-87052011005000001.

Simpson, C. E. and Starr, J. L. (2001). Registration of 'Coan' peanut. CropScience, 41, 918. https://doi.org/10.2135/cropsci2001.413918x.

Singh, A. K. and Simpson, C. E. (1994). Biosystematics and genetic resources. In J. Smartt (Ed.), The Groundnut Crop. (p. 96-137). London: Chapman \& Hall. https://doi.org/10.1007/978-94-011-0733-4_4.

Valls, J. F. M. and Pizarro, E. A. (1994). Collecting wild Arachis Germplasm. In: P. C. Kerridge, B. Hardy (Ed.), Biology and agronomy of forage Arachis (p. 19-27). Cali: CIAT.

Valls, J. F. M. (2005). Recursos genéticos de Arachis: Avanços no conhecimento botânico e a situação atual de conservação e uso. Agrociencia, 9, 123-132.

Valls, J. F. M. and Simpson, C. E. (2005). New species of Arachis (Leguminosae) from Brazil, Paraguay and Bolivia. Bonplandia, 14,35-64.

Valls, J.F.M., Costa, L.C. and Custodio, A.R. (2013). A novel trifoliolate species of Arachis (Fabaceae) and further comments on the taxonomic section Trierectoides. Bonplandia, 22, 91-97.

Veiga, R. F. A., Valls, J. F. M. and Lopes, C. R. (1992). Identification key to Brazilian populations of wild peanut, Arachis sylvestris (A.Chev.) A.Chev. Plant Genetic and Resources Newsletter, 107, 23-27.

Veiga, R. F. A., Valls, J. F. M., Lopes, C. R., Curi, P. R. and Krapovickas, A. (1999). Caracterização morfológica e agronômica de populações de Arachis sylvestris (A.Chev.) A.Chev. Boletim Científico, 47. Campinas: IAC.

Veiga, R. F. A., Voltan, R. B. Q., Valls, J. F. M., Fávero, A. P. and Barbosa, W. (2001). Caracterização morfológica de acessos de germoplasma de quatro espécies brasileiras de amendoim silvestre. Bragantia, 60,167-176. https://doi.org/10.1590/s0006-87052001000300003.

Veiga, R. F. A., Valls, J. F. M., Tombolato, A. F. C., Barbosa, W. and Pires, E. G. (2003). Amendoins silvestres para uso ornamental. Revista Brasileira de Horticultura Ornamental, 9, 7-15. https://doi. org/10.14295/rbho.v9i1.160. 